\title{
Visualisation of Time-Variant Respiratory System Elastance in ARDS Models
}

\author{
van Drunen $\mathrm{EJ}^{1}$, Chiew $\mathrm{YS}^{1}$, Zhao $Z^{2}$, Lambermont $\mathrm{B}^{3}$, Janssen $\mathrm{N}^{4}$, Pretty $\mathrm{C}^{5}$, Desaive $\mathrm{T}^{5}$, Moeller $\mathrm{K}^{2}$, Chase $\mathrm{JG}^{1}$ \\ ${ }^{1}$ Department of Mechanical Engineering, University of Canterbury, New Zealand \\ ${ }^{2}$ Institute of Technical Medicine, Furtwangen University, Germany \\ ${ }^{3}$ Medical Intensive Care Unit, University Hospital of Liege, Belgium \\ ${ }^{4}$ Emergency Department, University Hospital of Liege, Belgium \\ ${ }^{5}$ GIGA-Cardiovascular Sciences, University of Liege, Belgium
}

erwin.vandrunen@pg.canterbury.ac.nz

\begin{abstract}
Model-based mechanical ventilation (MV) can be used to characterise patient-specific condition and response to $M V$. This paper presents a novel method to visualise respiratory mechanics during $M V$ of patients suffering from acute respiratory distress syndrome. The single compartment lung model is extended to monitor time-varying respiratory system elastance within each breathing cycle. Monitoring continuous in-breath mechanics allows changes to be observed continuously, providing more insight into lung physiology. Thus, this new monitoring method may potentially aid clinicians to guide $M V$ in a heterogeneous population.
\end{abstract}

Keywords: Respiratory mechanics, mechanical ventilation, ARDS

\section{Introduction}

Modelling the breath-to-breath respiratory mechanics of acute respiratory distress syndrome (ARDS) patients can potentially provide a non-invasive, patient-specific method to obtain clinically useful information in real-time to guide treatment $[1,2]$. However, this method of monitoring is limited in clinical application [2-5].

Dynamic respiratory system elastance $\left(E_{d r s}\right)$ is a breathspecific time-varying elastance $[6,7]$. This dynamic parameter within a single breath provides unique insight into a patient's breathing pattern, revealing lung recruitment and overdistension. In addition, identifying minimum $E_{d r s}$ also reveals the potential to titrate optimal patient-specific positive end-expiratory pressure (PEEP) to maximise recruitment without inducing lung injury [7]. This work presents a novel method in visualising $E_{d r s}$. Monitoring breath-to-breath time-variant $E_{d r s}$ can provide a higher resolution metric to guide MV therapy than existing respiratory mechanics monitoring.

\section{Methods}

\section{Dynamic Respiratory System Elastance Model}

The equation of motion describing the airway pressure as a function of the resistive and elastic components of the respiratory system is defined as:

$$
P_{a w}(t)=R_{r s} \times Q(t)+E_{r s} \times V(t)+P_{0}
$$

where $P_{a w}$ is the airway pressure, $t$ is time, $R_{r s}$ is the resistance of the conducting airway, $Q$ is the air flow, $E_{r s}$ is the respiratory system elastance (1/compliance), $V$ is the lung volume and $P_{0}$ is the offset pressure. $E_{r s}$ and $R_{r s}$ can be determined using multivariate regression or the integralbased method [7]. If $R_{r s}$ is assumed constant throughout a breath $[3,4,7], E_{d r s}$ can be re-estimated using Equation (2) after $R_{r s}$ is estimated using Equation (1).

$$
E_{d r s}(t)=\frac{P_{a w}(t)-P_{0}-R_{r s} \times Q(t)}{V(t)}
$$

Patient-specific $E_{d r s}$ is only analysed during the inspiration portion of the breathing cycle. Arranging each breathing cycles' $E_{d r s}$ curve such that it is bounded by the $E_{d r s}$ curve of the preceding breath and the subsequent breath leads to a three-dimensional, time-varying, breathspecific $E_{d r s}$ surface. This method of visualisation will give clinicians new insight into how respiratory mechanics change with time over the course of treatment.

\section{Lavage ARDS Animal Models}

A study was performed using experimental ARDS piglets. After intubation via tracheotomy, the piglets were ventilated using a Draeger Evita2 ventilator (Draeger, Lubeck, Germany). The ventilator was set to intermittent positive pressure ventilation mode (IPPV) to deliver a tidal volume of $8-10 \mathrm{ml} / \mathrm{kg}$, with a $\mathrm{FiO}_{2}$ of 0.5 , at 20 breaths $/ \mathrm{min}$. The subjects underwent repeated lavage to induce ARDS. The arterial blood gases were monitored and once diagnosed with ARDS $\left(\mathrm{PaO}_{2} / \mathrm{FiO}_{2}<200 \mathrm{mmHg}\right)$, each subject underwent a staircase recruitment manoeuvre (RM) with PEEP settings at 1-5-10-15-20-15-10-5-1 mbar. Each PEEP level was maintained for 10-15 breaths before changing to the next PEEP level. For the duration of the $\mathrm{RM}$, a visage manœuvre was maintained to ensure an adequate level of seriousness. Airway pressure and flow were measured using a 4700B pneumotachometer (Hans Rudolph Inc., Shawnee, KS).

\section{Results}

Figure 1 shows two examples of the $E_{d r s}$ surface for the lavage ARDS piglets. The top surface shows the $E_{d r s}$ for every breathing cycle. The change in airway pressure 
from PEEP to peak inspiratory pressure (PIP) is shown in grey at the bottom of each plot.
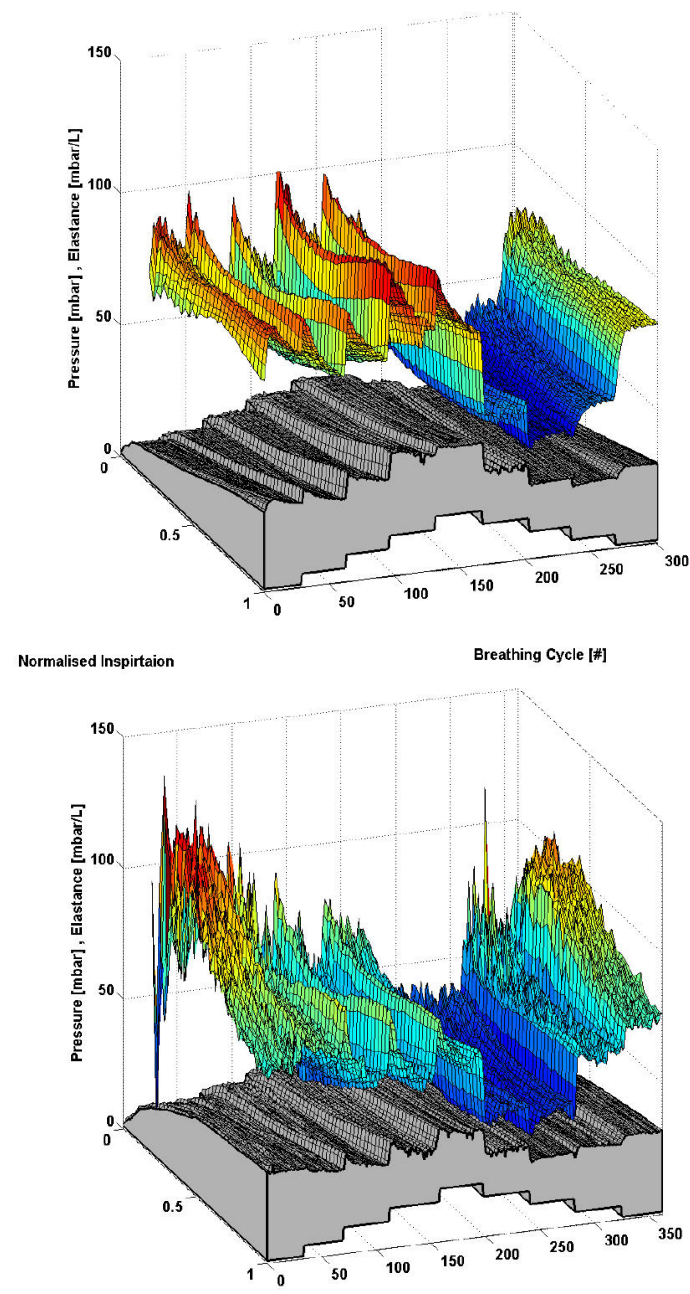

Normalised Inspirtaion

Breathing cycle [*]

Figure 1: $E_{d r s}$ surface across a normalised breath during a RM for two lavage ARDS piglets.

\section{Discussion}

Immediately after a PEEP increase, the $E_{d r s}$ trajectory descends to a minimum before once again increasing towards the end of inspiration. Rising $E_{d r s}$ at the end of inspiration indicates a potential for lung damage due to overstretching which may not be captured by a single value of $E_{r s}$. However, as PEEP is maintained for a few breaths, it was found that each successive breath has a reduced $E_{d r s}$ rise, indicating a period of stabilisation and a time-dependency of respiratory elastance and viscoelastic properties, reducing the hysteresis.

The $E_{d r s}$ surface for both ARDS piglets shows a significant reduction in respiratory elastance during decreasing PEEP titration. It was found that global minimal elastance PEEP occurs at 10 mbar. During a PEEP increase, $E_{d r s}$ decreases as recruitment of new lung volume outweighs lung stretching [7]. It was found that $E_{d r s}$ during increasing PEEP is significantly different to $E_{d r s}$ during decreasing PEEP $(p<0.005)$. Thus, this case shows the typical finding where PEEP should be titrated after the lung has been recruited $[3-5,7]$. However, a local minimum $E_{d r s}$ was also found at 10 mbar during increasing PEEP titration, suggesting similar optimal PEEP settings.

Selecting PEEP is a trade-off in minimising lung pressure and potential damage, versus maximising recruitment. In addition, recruitment is also a function of PEEP and time [8]. Therefore, true minimal $E_{d r s}$ can only be determined after a stabilisation period is provided at each PEEP level. Setting PEEP at minimum elastance theoretically benefits ventilation by maximising recruitment, reducing work of breathing and avoiding overdistension [3-5, 9].

The time-varying $E_{d r s}$ is a higher resolution metric of dynamic adaptation to PEEP than a single elastance value, $E_{r s}$, used in existing clinical practice. Thus, real-time monitoring of $E_{d r s}$ can potentially guide decision making in the intensive care unit. Changes in ventilator mode to modify the $E_{d r s}$ surface could also be used to guide therapy.

\section{Bibliography}

[1] A. Sundaresan and JG. Chase, "Positive end expiratory pressure in patients with acute respiratory distress syndrome - The past, present and future," Biomedical Signal Processing and Control, vol. 7, pp. 93-103, 2011.

[2] L. Brochard, G. Martin et al., "Clinical review: Respiratory monitoring in the ICU - a consensus of 16," Crit Care, vol. 16, p. 219, 2012.

[3] A. Carvalho, F. Jandre et al., "Positive end-expiratory pressure at minimal respiratory elastance represents the best compromise between mechanical stress and lung aeration in oleic acid induced lung injury," Crit Care, vol. 11, p. R86, 2007.

[4] F. Suarez-Sipmann, S. H. Bohm et al., "Use of dynamic compliance for open lung positive end-expiratory pressure titration in an experimental study," Crit Care Med, vol. 35, pp. $214-221,2007$.

[5] B. Lambermont, A. Ghuysen et al., "Comparison of functional residual capacity and static compliance of the respiratory system during a positive end-expiratory pressure (PEEP) ramp procedure in an experimental model of acute respiratory distress syndrome," Crit Care, vol. 12, p. R91, 2008.

[6] C. A. Stahl, K. Moller et al., "Dynamic versus static respiratory mechanics in acute lung injury and acute respiratory distress syndrome," Crit Care Med, vol. 34, pp. $2090-2098,2006$.

[7] Y. S. Chiew, JG. Chase et al., "Model-based PEEP optimisation in mechanical ventilation," BioMedical Engineering OnLine, vol. 10, p. 111, 2011.

[8] S. P. Albert, J. DiRocco et al., "The role of time and pressure on alveolar recruitment," Journal of Applied Physiology, vol. 106, pp. 757-765, March 20092009.

[9] Z. Zhao, D. Steinmann et al., "PEEP titration guided by ventilation homogeneity: a feasibility study using electrical impedance tomography," Crit Care, vol. 14, p. R8, 2010. 